\title{
Synthesis of 'Tracers Using Automated Radiochemistry and Robotics
}

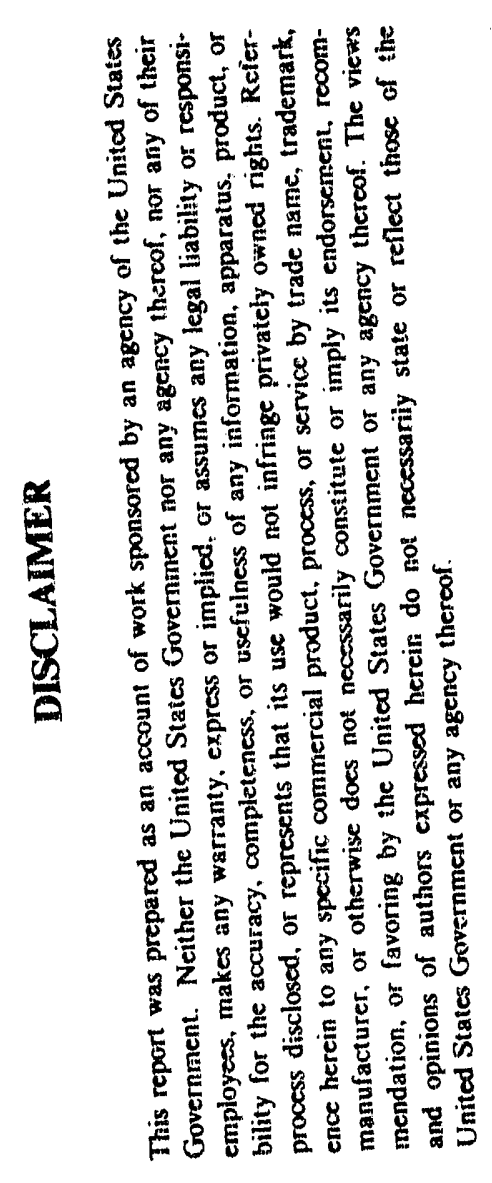

\author{
Progress Report \\ for Period April 1, 1992 - July 31, 1992 \\ Robert F. Dannals, Ph.D. \\ The Johns Hopkins University \\ School of Medicine \\ Division of Nuclear Medicine \\ Baltimore, Maryland 21205-2179 \\ Report Date: July 1992

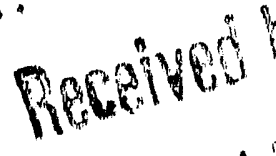

Prepared for:

The United States Department of Energy

Grant Number DE-FG02-90ER61006

\section{MASTER}


R. F. Dannals, Ph.D.

\section{Proposal Abstract}

The synthesis of high specific activity radiotracers labeled with short-lived positron-emitting radionuclides for use in positron emission tomography (PET) often requires handling large initial quantities of radioactivity for a successful tracer preparation and PET study. High specific activities are required when preparing tracers for use in PE'T studies of neuroreceptors, which are small biomacromolecular recognition sites that are finite in number and easily saturated. With the current demands for production of radiotracers for PET, a fully automated approach for tracer synthesis is highly desirable. This proposal involves the development of a system for the Synthesis of Tracers using Automated Radiochemistry and Rojotics (STARR) for this purpose.

While the long range objective of the proposed research is the development of a totally automated radiochemistry system for the production of major high specific activity ${ }^{11} \mathrm{C}$-radiotracers for use in PET, the specific short range objectives of the proposed research are the automation of ${ }^{11} \mathrm{C}$-methyl iodide $\left({ }^{11} \mathrm{CH}_{3} \mathrm{l}\right)$ production via an integrated approach using both radiochemistry modular labstations and robotics, and the extension of this automated capability to the production of several radiotracers for PET (initially, ${ }^{11} \mathrm{C}$-methionine, 3-N-[11C-methyl]spiperone, and [ $\left.{ }^{11} \mathrm{C}\right]$-carfentanil).

Keywords: radiochemistry, PET, robot, radiotracer, radiopharmaceutical 
R. F. Dannals, Ph.D.

\section{Summary of Progress}

All present results are consistent with the overall goal of the project to develop a system for the synthesis of tracers using automated radiochemistry and robotics. The research supported by this grant supports the DOE mission through its development of remote methods of handling radioactive materials, with special emphasis on robotics and automated radiochemistry technology development, for the radiochemical synthesis of positron emitting tracers for application to problems of public health. The research directly impacts on the DOE mission in that the development of positron emission tomography and related technology currently supported by DOE research is necessary for continued progress in labeled compound development, biomedical research, and clinical application of PET.

Due to some uncertainty in funding of the final nine months of this project, this report represents only the first 3 months of the final year of the project. The uncertainty in funding caused a delay in initiating the final research objective - an automated adaptive HPLC monitoring system. Since funding of this research began in August 1990, an automated modular labstation for ${ }^{11} \mathrm{CH}_{3} \mathrm{I}$ production was constructed, installed, interfaced to the robot, and the robot control system was programmed. The radiochemical yield, radiochemical purity, and specific activity of ${ }^{11} \mathrm{CH}_{3}$ I produced by the automated modular labstation was determined.

During the current funding period, a new faster robotic arm (Zymark Zymate XP) was further tested. The faster arm minimized the delay in moving air sensitive reagents and reaction vessels during the radiochemical synthesis. With the faster robot, a new robot control system was required. This has required additional debugging of the automated modular labstation. However, as a result of the additional precautions taken to minimize carrier carbon contamination, the average specific activity at the end of methyl iodide production has increased to $3515 \pm 279$ $\mathrm{mCi} / \mu$ mole (determined ty UV standard curves).

At the present time, the tracers which remain to be synthesized require high specific activity. Although $3-\mathrm{N}-\left[{ }^{11} \mathrm{C}-\right.$-methyl] spiperone and [ $\left.{ }^{1} \mathrm{C}\right]$ carfentanil have been purified using "timed" HPLC separations (i.e., averaging elution times from several manual syntheses and collecting the

Page: 3 
- eluant based solely on the averaged times), the variability in elucion times is great enough to limit the widespread use of this method. An "intelligent" on-line HPLC monitoring program which will adjust collection parameters according to observed UV and radiochromatographic signals from detectors is under development. These chromatographic developments will be used in the syntheses of [ $\left.{ }^{11} \mathrm{C}\right]$ carfentanil and 3-N-[1"C-methyl] spiperone.

The high specific activity "C-methyl iodide produced above was used in the robotic synthesis of 3-N-[11 C-methyl]spiperone and most recently, $\left[{ }^{11} \mathrm{C}\right]$ carfentanil, using STARR. The average synthesis times are approximately double those obtained manually. The average specific activity at end-of-synthesis from STARR is approximately $1875 \mathrm{mCi} / \mu \mathrm{mole}$ (compared to vver $2500 \mathrm{mCi} / \mu$ mole when the synthesis is performed manually). The increase in synthesis time is attributed in part to the use of slower flow rate on the HPLC semipreparative purification (the slower flow rate appears to increase the reliability of the timed collections of product in the HPLC eluant). The increase in the synthesis time is also attributed to the lack of a suitable means for determining the endpoint in the evaporation of the HPLC solvent from the collected eluant.

The current research involves the development of an "intelligent" on-line HPLC monitoring program which will adjust collection parameters according to observed UV and radiochromatographic signals from detectors. 

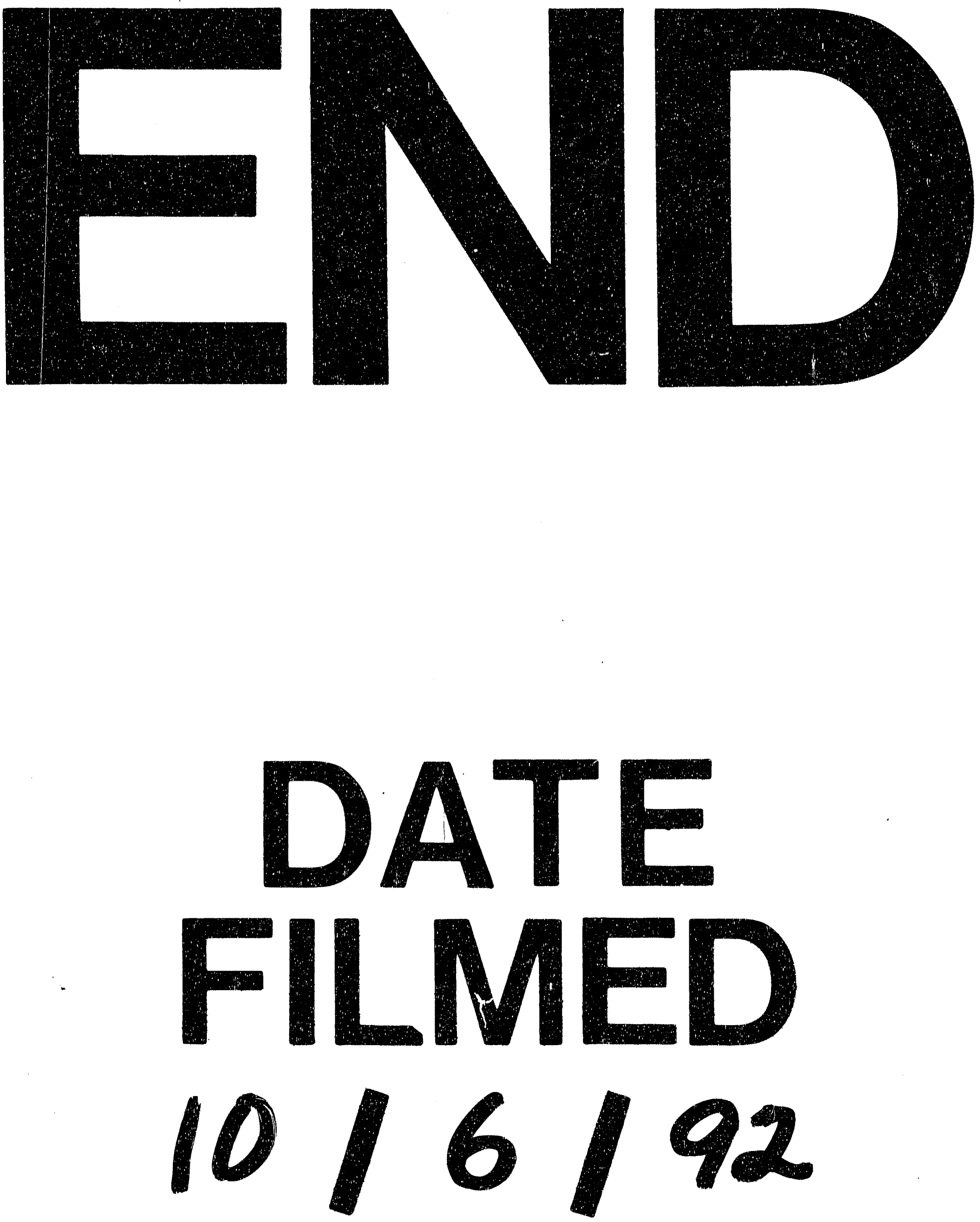

1 


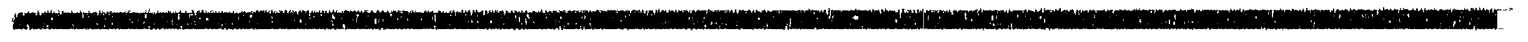

